be of the greatest value. The readers of NATURE will appreciate anything that helps the scientific worker. Now, at last, we are going to the root of things in biology, and only the embryologist knows fully what a boon an egghatcher, convenient and easy to work, would be. The eggs of the hen will be wanted in their various stages of incubation, as long as there are workers in these departments, but many other sorts of oviparous animals have to be worked out in all their stages besides the common fowl. Snakes, lizards, tortoises, crocodiles, all these are rivals of the bird in their embryology, and of many kinds the eggs could be procured and their embryos developed if the worker had some such apparatus as $\mathrm{Mr}$. Christy is bringing out. We want, not merely the genera] embryology of these ovipara, such as is so excellently illustrated and described in Messrs. Foster and Balfour's work, but the special development of any important organ ought to be traced in all its stages through not one, but many types of the vertebrata: through all the principal kinds indeed.

Some of us are trying to do this in the skeletal structures; the nervous system, still more important, wants an army of workers, then there are the respiratory, digestive, excretory, and generative organs, all these want a complete history in all their stages, not in one type merely, but in scores of types. We therefore wish well to all energetic and enterprising men who put it into our power to work on a wider scale; such means and appliances as can be brought out by men too restless for close and patient study, may be of infinite service to the close and patient student, who is too dreamy and abstracted to invent for himself.

W. K. P.

\section{NOTES ON SOME NATAL PLANTS}

GROWING plentifully among the grass on the coast hills of Natal is a small blue flower belonging to the Rubiaceæ. In this plant, generally speaking, there are two forms only, in one of which the five stamens are exserted considerably beyond the tube of the rotate corolla, and the stigma is included in the tube; in this form the tube is almost devoid of hairs. In the other common form the position of these essential organs is reversed, the stigma protruding to about the same extent that the stamens do in the first mentioned, and the stamens being included; here, however, the upper part of the corolla tube is thickly covered with downy hairsof course this is an ordinary dimorphic plant. But I find lately a third form of the same species (only, however, rarely) in which both stamens and stigma are exserted and are of the same length, so that here self-fertilisation must take place, as the stamens and stigma touch at the time the former dehisce. I do not think this can be termed a cleistogamic form, as, although rather smaller and lighter in colour than the others, the difference is only trifling. The hairs which cover the corolla-tube in the form with included stamens serve to keep the pollen collected near the upper part of the tube, as, if it fell to the base it would not be so easily transferred by the proboscis of an insect as when lightly held by the hairs through which the insect must make way. As these hairs would be for this purpose useless when the stamens are exserted they do not occur in the other form.

I notice the same arrangement of hairs in another dimorphic plant belonging, I think, to the same order, which grows on the marshy flats near the sea. I have found on the coast lands here four other plants, in which cross-fertilisation is secured by dimorphism, one of them being a monocotyledonous plant.

There is a species of Polygonum which climbs in the bush which well illustrates another plan ensuring crossfertilisation; while the flower is young and the perianth still closed, enveloping the immature stamens, the three branching stigmas protrude from between the segments in a fit state to receive the pollen. If (as is usual) the ripe stigmas were only exposed when the flower opens, although the evils of self-fertilisation would of course be avoided by the plant being proterogynous, still, as it is wind-fertilised, the perianth and stamens would be in the way of any stray pollen-grains reaching the stigmas; while as it is, nothing interposes between pollen and stigmas.

Lately I have found a curious aberration of form in Tecoma capense growing here. It is very common in the bush, forming great beds of bright colour, and normally has a scarlet trumpet-shaped corolla, with one rudimentary and four perfect stamens. I found, however, three or four plants growing within a short distance of each other, in which there were eight perfect stamens; they seemed, however, to have been formed at the expense of the corolla, for there was only one segment coloured at all, the remainder being colourless and small. The ovary seemed in several cases to have been fertilised. The ordinary form of this plant, although individually so brightly coloured, growing in large numbers and secreting much nectar, is seldom or never visited by Lepidoptera. It is, however, frequented by honeysuckers and small bees in numbers. All through the day you can hear the shrill chirp of the small bright honeysucker among the blossoms. The immediate reason why butterflies and moths do not visit it I cannot give ; but the stamens and stigma (which are beneath the large upper segment of the corolla) are long, and so high above the opening of the corolla-tube that those insects, in visiting the flower for its nectar, would not be at all certain to touch either, and so in comparison to the honeysucker and small bees would be of little benefit to the plant; for when the former of these visits the flower the feathers of his head are just of the height to brush off the pollen, and the latter in collecting the pollen is equally certain to distribute it, as the bifid stigma is about the same length or only slightly longer than the stamens. Can the nectar have been modified to suit the taste of the useful honeysucker without reference to the useless butterfly?

Natal, June 27

$$
\text { M. S. Evans }
$$

\section{PHYSICS IN PHOTOGRAPHY}

III.

THESE last experiments were remarkable in another point of view, as they opened out the question as to whether the salts of silver might not prove sensitive to rays to which they had been supposed hitherto to be insensitive. Silver iodide, for instance, when exposed to the spectrum in a solution of potassium sulphite proved sensitive as far as " $a$ " of the spectrum instead of stopping short at the point indicated in Fig. 2 (p. 529); and silver bromide in the molecular grouping which absorbed the red proved sensitive to a wave-length of somewhere near I 1,000 , whereas in its normal state 9,600 was its limit.

Similarly silver chloride proved sensitive to an extent which presumably may be increased till it is equal to that of the bromide. In both these instances we have a proof that the compound was sensitive to these abnormal rays, and that the image formed by those rays was destroyed as soon as formed by their oxidising action giving an undevelopable form of salt. It may be remarked that by exposing films in reducing solutions such as ferrous sulphate, and pyrogallic acid rendered very slightly alkaline, that an image can be developed as fast as it is formed.

The natural outcome of the experiments on the oxidation of the photographic image just narrated is that it should lead to the solution of the problem of photom graphy in natural colours, such as that of Becquerel, Niepce de St. Victor, and others. In the fourth edition

$$
\therefore \text { Continued from p. } 53^{x} \text {. }
$$

